\title{
Efficient Rectilinear Steiner Tree Construction with Rectilinear Blockages
}

\author{
Zion Shen \\ Cadence Design Systems \\ 555 River Oaks Parkway \\ San Jose, CA, 95134 \\ zion@cadence.com
}

\author{
Chris C.N. Chu
}

\author{
Department of ECE \\ Iowa State University \\ Ames, Iowa, 50011 \\ cnchu@iastate.edu
}

\author{
Ying-Meng Li \\ Atoptech, Inc. \\ 2700 Augustine Drive \\ Santa Clara, CA 95054
}

\begin{abstract}
Given $n$ points on a plane, a Rectilinear Steiner Minimal Tree $(R S M T)$ connects these points through some extra points called steiner points to achieve a tree with minimal total wire length. Taking blockages into account dramatically increases the problem complexity. It is extremely unlikely that an efficient optimal algorithm exists for Rectilinear Steiner Minimal Tree Construction with Rectilinear Blockages (RSMTRB). Although there exist some heuristic algorithms for this problem, they have either poor quality or expensive running time.
\end{abstract}

In this paper, we propose an efficient and effective approach to solve RSMTRB. The connection graph we used in this approach is called spanning graph which only contains $O(n)$ edges and vertices. An $O(n \log n)$ time algorithm is proposed to construct spanning graph for RSMTRB. The experimental results show that this approach can achieve a solution with significantly reduced wire length. The total run time increased is negligible in the whole design flow.

\section{INTRODUCTION}

Given $n$ points on a plane, a Rectilinear Steiner Minimal Tree $(R S M T)$ connects these points through some extra points called steiner points to achieve a tree with minimal total wire length. Many works [1-12,14-17] have been done on this fundamental problem in electronic design automation. However, most of them did not take blockages into consideration. In fact, today's design often contains many rectilinear routing blockages, e.g., macro cells, IP blocks, and pre-routed nets. Thus, rectilinear Steiner minimal tree construction with rectilinear blockages $(R S M T R B)$ becomes a very practical problem.

Generally, $R S M T$ is used in initial net topology creation for global routing or incremental net tree topology creation in physical synthesis. It is also utilized to estimate total wire length, congestion and timing in early design stages, like block floorplanning and cell placement. The timing and congestion information obtained from $R S M T$ can be used as a criteria in following timing and congestion driven

Permission to make digital or hard copies of all or part of this work for personal or classroom use is granted without fee provided that copies are not made or distributed for profit or commercial advantage and that copies bear this notice and the full citation on the first page. To copy otherwise, to republish, to post on servers or to redistribute to lists, requires prior specific permission and/or a fee.

Copyright 2005 ICCD X-XXXXX-XX-X/XX/XX ...\$5.00. routing. Note that it is the problem applied hundreds of thousands times in each design flow and many times this problem comes with very large input size. $R S M T$ thus deserves much intensive research in VLSI CAD.

Unfortunately, RSMT itself was first shown to be strongly NPcomplete by Garey [7] in 1977. Taking blockages into account dramatically increases the problem complexity. Thus, it is extremely unlikely that an efficient optimal algorithm exists for $R S M T R B$. Although there exist some heuristic algorithms for this problem, they have either poor quality or expensive run time.

Existing heuristics for $R S M T R B$ can be classified into three categories.

The first category is maze routing [8] based approach. Maze routing can optimally route two pin nets. However, for multi-pin nets, designers need to introduce a multi-terminal variant [9-11], which incurs a solution far from optimal. In addition, since the run time and memory used in maze routing are proportional to the size of the routing area rather than the size of actual problem (i.e., the number of pins and blockages), maze routing algorithms are inefficient in terms of run time and memory.

The second category is called sequential approach which typically consists of two steps. These two steps are illustrated in Figure 1. Step 1 is to construct a tree $T_{1}$, which is either a minimum spanning tree $(M S T)$ or a Steiner minimal tree $(S M T)$ with absence of blockages. In this example, $T_{1}$ is an $M S T$. Step 2 is to transform $T_{1}$ to a $R S M T$ with blockages by substituting edges around the blockages for the edges overlapped by the blockages. Generally a simple line sweep technique is applied in step 2. For example, for pin pair $\left(p_{1}, p_{4}\right)$, we have two possible L-shaped routes, $r_{1}$ and $r_{2}$ to interconnect $p_{1}$ and $p_{4}$ as illustrated in Figure 2(a) with absence of blockages. Then we sweep all blockages which overlap with these two routes and choose one route with smaller corresponding detour. As shown in Figure 2(b), route $r_{2}$ are selected since the detour length introduced by $r_{2}$ is smaller than that by $r_{1}$. This approach is commonly used in industry due to its simplicity and efficiency. However, since step 1 neglects the global view of blockages, step 2 can only locally remove overlap between $T_{1}$ and blockages. The quality(i.e., the total wire length) of resulting $R S M T$ can be much worse than expected in many cases.

Later on, Yang et.al [12] introduced a complicated 4-process heuristics to remove the overlaps in step 2 in a clever way. However the approach still cannot avoid bad solution for many cases. This is because a bad $T_{1}$ due to neglecting blockages in step 1 could introduce an unexpected routing detour in step 2 .

The third category consists of connection graph [13] based approaches. Typically, the approach in this category is to first construct a connection graph by pins and blockage boundaries, which guarantees that at least one rectilinear Steiner minimal (or close to minimal) tree is embedded in the graph. Then, some graph searching tech- 


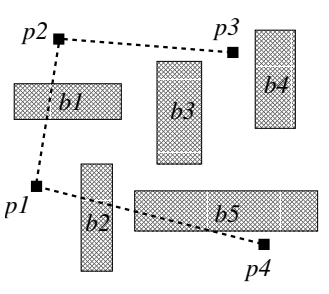

Step 1

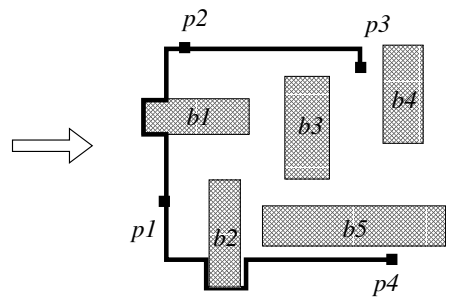

Step 2
Figure 1: Sequential approach to solve $R S M T R B$.

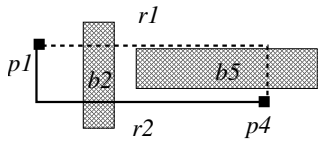

(a)

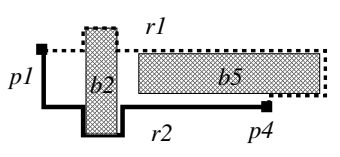

(b)
Figure 2: Overlap removal in sequential approach. (a) two Lshaped routes, $r_{1}$ and $r_{2}$, before overlap removal. (b) route $r_{1}$ and $r_{2}$ after overlap removal.

nique is used to find a $R S M T$ as a subgraph from the connection graph. Unlike sequential approach, this approach can globally catch the view of both pins and blockages in the design. Therefore, the connection graph based approach can generally achieve an optimal or near optimal $R S M T$.

The efficiency of connection graph based approach depends on the size of graph. While the accuracy or effectiveness of this approach depends on whether the graph contains a good Steiner minimal tree. Obviously, there is a trade-off between efficiency and accuracy in this approach.

In [14], a connection graph is called escape graph which is constructed by escape segments. The number of vertex in the escape graph can be $O\left(n^{2}\right)$ in the worst case, where $n$ is the sum of pins and blockage boundaries. Even the size of a reduced escape graph is still quite huge. In [15], authors proposed a connection graph with $O(n \log n)$ vertices and edges and later on [16], they introduced an even smaller graph which contains $O(n \sqrt{\log n})$ vertices and $O\left(n \log ^{3 / 2} n\right)$ edges. The construction of the connection graph takes $O\left(n \log ^{3 / 2} n\right)$ time and memory usage.

In this paper, we will propose an efficient and effective connection graph. It is called spanning graph. We show that the spanning graph contains only $O(n)$ vertices and $O(n)$ edges, which is smaller than any previous connection graph. In addition, we show that our spanning graph can always produce a $R S M T$ with good quality. Due to the special property of the spanning graph, the construction takes only $O(n \log n)$ time and memory usage, which are also smaller than those in any previous connection graph construction.

We organize the rest of the paper as follows. In section 2 , we will formally define the problem and explain the details of the basic component in the problem. In section 3, we will first demonstrate the drawback in the escape graph and then introduce the spanning graph as a connection graph in $R S M T R B$. Section 4 and 5 describe the details of construction of spanning graph for $R S M T R B$. The experimental results are shown in section 6 . The paper is concluded in section 7 .

\section{PROBLEM FORMULATION}

Let $P=\left\{p_{1}, p_{2}, p_{3}, \ldots, p_{m}\right\}$ be a set of pins for $m$ pin net. Let $B=\left\{b_{1}, b_{2}, b_{3}, \ldots, b_{k}\right\}$ be a set of rectangular blockages. Let $V=\left\{v_{1}, v_{2}, v_{3}, \ldots, v_{n}\right\}=P \cup\{$ corners in $B\}$ as the vertex set in the problem, where each $v_{i}$ has coordinates $\left(x_{i}, y_{i}\right)$. Note that each rectangular blockage has 4 corners, we have $n \leq m+4 k$. The

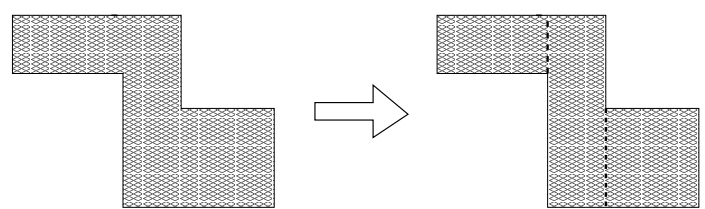

Figure 3: Dissect a rectilinear blockage into 3 rectangular blockages.

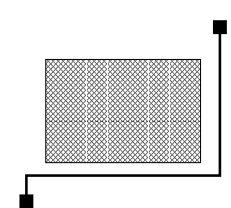

(a)

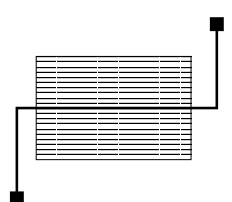

(b)

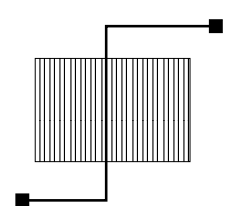

(c)
Figure 4: Three types of blockages: (a) Complete blockage (b) Vertical blockage (c) Horizontal blockage

rectilinear distance between $v_{i}$ and $v_{j}$ is given as $\left|x_{i}-x_{j}\right|+\mid y_{i}-$ $y_{j} \mid$. A $R S M T$ connects all pins through some extra points (called Steiner points) to achieve a minimal total length, while avoiding the intersection with any blockage in the design.

\subsection{Rectilinear blockages}

If all boundaries of a blockage are either horizontal or vertical, we call this blockage as rectilinear blockage. Note that each rectilinear blockage can be dissected into a set of rectangular blockages (see Figure 3 as an example). In the rest of paper, for simplicity, we assume each blockage to be rectangular.

\subsection{Directional blockages}

In multi-layer routing, there exist three types of blockages. The first one is called complete blockage which blocks all vertical and horizontal metal layers in its obstructed area. A complete blockage requires all routes must detour around it. The second type is denoted as vertical blockage in which all vertical layers in obstructed area are blocked while a certain number of horizontal layers are still available for routing. The routes are allowed to horizontally pass through the obstructed area, while not allowed in vertical direction. The third type is called horizontal blockage, where routes can still vertically pass through the obstructed area. These three types of directional blockages are illustrated in Figure 4.

\section{ESCAPE GRAPH VS. SPANNING GRAPH}

\subsection{Redundancy in escape graph}

As we mentioned in Section 1, a connection graph can catch the global view of both blockages and pins. And the efficiency of this approach highly depends on the size of the connection graph. In other words, a good connection graph is able to describe all necessary geometrical relationship between pins and blockages using as few edges as possible. In [14], the connection graph is called escape graph, which is constructed by escape segments. Escape segments are formed by horizontal and vertical lines extending from pins and blockage boundaries, and ending with their abutment to either a blockage boundary or the internal perimeter of the routing region. The number of vertex in the escape graph can be $O\left(n^{2}\right)$ in the worst case. An example is shown in Figure 5. The collection of the escape segments (shown as dashed segments) composes the escape graph. And the graph preserves a good $R S M T$ for a multi-pin net. However, we notice that most of edges and vertices are redundant in the escape graph for finding a $R S M T$. For example, instead of using 


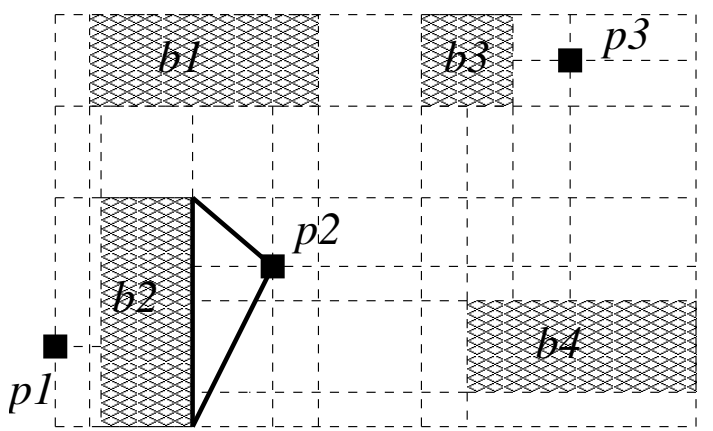

Figure 5: Escape graph

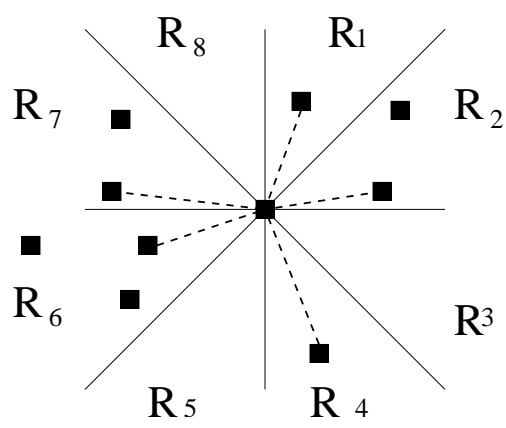

Figure 6: Eight regions defined for each point in spanning graph.

13 edges, 3 edges (shown as solid segments) are enough to represent the connection relationship between the blockage $b_{2}$ and pin $p_{2}$ in the corresponding connection graph.

\subsection{Spanning graph}

In [17], a spanning graph is introduced as an intermediate step in minimal spanning tree construction. Given a set of points on the plane, a spanning graph is an undirected graph over the points that contains at least one minimal spanning tree. The number of edges in the graph is called the cardinality of the graph.

The construction of spanning graph is illustrated in Figure 6. From each point $p$, a plane can be divided into 8 regions by horizontal, vertical and $\pm 45^{\circ}$ lines through $p$. It can be proved that the rectilinear distance between any two points in one region is always smaller than the maximal distance from them to $p$. Due to the cycle property of a minimal spanning tree, that is, the longest edge on any cycle should not be included in any minimal spanning tree, which means only the closest point to $p$ in each region needs to be connected to $p$. Considering all given points, the connections will form a spanning graph of cardinality $O(n)$. In other words, spanning graph is able to describe the relative geometrical relationship between points in the plane using $O(n)$ edges.

Enlightened by the spanning graph in $M S T$ construction, we borrow and revise this idea to solve $R S M T R B$. The details are presented in following section.

\section{SPANNING GRAPH BASED APPROACH IN $R S M T R B$}

\subsection{Search regions}

In general spanning graph, each point $p$ corresponds to 8 regions which are divided by the horizontal, vertical and $\pm 45^{\circ}$ lines going through $p$. Then we search each region and find the closest point in each region and connect it to $p$. While in $R S M T R B$, for each blockage $b_{i}$, we divide the whole plane into 8 regions as shown in Figure $7(a)$. Each corner of blockage $b_{i}$ has 3 neighboring search

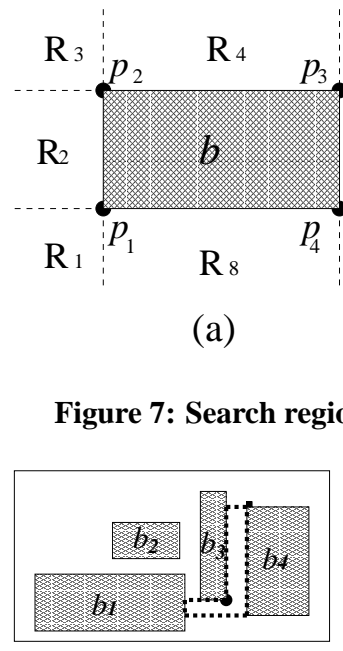

(a)

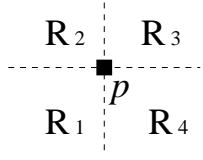

(b)
7: Search regions for blockage and pin

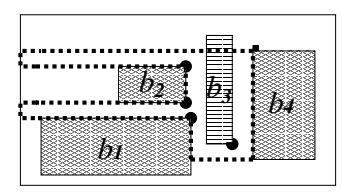

(b)
Figure 8: Visible points in search region for different blockages

regions which are adjacent to this corner. We call these three regions as neighboring search regions for a given corner point. For example, the neighboring search regions for the lowerleft corner $p_{1}$ in Figure $7(a)$ are $R_{8}, R_{1}$ and $R_{2}$. For each pin $p$, we divide the whole plane into 4 search regions. The corresponding neighboring search regions for $p$ are $R_{1}, R_{2}, R_{3}$ and $R_{4}$ as shown in Figure 7(b).

For each $v \in V$, we connect the closest visible point in $v$ 's each neighboring search region to $v$. Note that the visible point in each search region could be different for complete blockage and directional blockage. For example, between Figure 8(a) and Figure 8(b), the only difference is that the blockage $b_{3}$ is a complete blockage in Figure $8(a)$ while a vertical blockage in Figure $8(b)$. The search region $R_{2}$ for blockage $b_{4}$ is denoted as the area enclosed by the dashed segments. Obviously, the search region in Figure $8(b)$ is larger than that in Figure 8(a). In Figure 8(a), there exists only 1 visible point in region $R_{2}$ of $b_{4}$. While in Figure $8(b)$, there are 4 visible points in $R_{2}$ of $b_{4}$.

A few natural questions may be raised as follows. First, why do we not apply $\pm 45^{\circ}$ lines in defining $R S M T R B$ search region? If $\pm 45^{\circ}$ lines are applied, each corner and pin has 6 and 8 search regions, respectively. We find that in practice, the total number of edges for spanning graph by our method is actually very close to the one by the method where $\pm 45^{\circ}$ lines are applied. However, ignoring these $\pm 45^{\circ}$ lines can greatly simplify the construction of spanning graph.

Second, why do we only consider 3 neighboring search regions for each corner $p$ of blockage $b$ ? This is because for any visible point $q$ in the rest 5 search regions of $b$, we can always find another corner $p^{\prime}$ of $b$ such that $q$ lies in one search region of $p^{\prime}$ and a shortest path from $p$ to $q$ can always be obtained by making $p^{\prime}$ as an intermediate point in the path.

In addition, for each point $p$, why do we only consider the connection with its visible points? The reason is that between the visible region and invisible region, there must exist at least one intermedi-

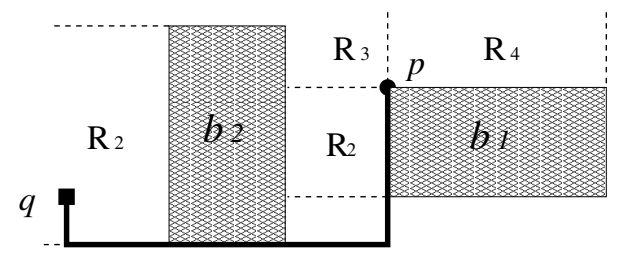

Figure 9: Example of invisible region for point $p$ 
ate blockage such that the point $p$ can always reach the points in invisible region by making use of the boundaries of the intermediate blockage(s) as part of the shortest path. As an example in Figure 9, region $R_{2}$ of blockage $b_{2}$ is invisible to the upperleft corner $p$ of $b_{1}$. However, any point in $R_{2}$ of $b_{2}$ can be reached from $p$ by using boundaries of $b_{2}$ as part of the shortest path.

\subsection{Spanning graph construction in $R S M T R B$}

First, from blockage set $B$, we find out a subset $B_{s}$ which includes each blockage lying within or intersected by the bounding box of net. Let $V_{s}$ be a point set which includes all corners of $B_{s}$ and pins of a given net. Our initial spanning graph $G$ has $V_{s}$ as its vertices and all blockage boundary segments of $B_{s}$ as its edges. Then, we incrementally build the graph by applying a sweep line based edge connection among vertices in $V_{s}$. For any point $v \in V_{s}$, we only connect at most one visible point $v^{\prime}$ in each neighboring search region to $v$, where $v^{\prime} \in V_{s}$ and $v^{\prime}$ is the closest point to $v$ in the corresponding neighboring search region.

Based on an $O(n \log n)$ sweep line algorithm proposed in [17], we propose a revised sweep line algorithm to construct spanning graph in $R S M T R B$. Our construction algorithm consists of four passes. Each pass performs edge connection for a pair of search regions of $V_{s}$. For sake of simplifying the exposition, we only present the detail procedures of Pass one which performs edge connection for search region $R_{2}$ and $R_{6}$ of all corner points.

First, all points in $V_{s}$ are sorted by their $x$ coordinates in nondecreasing order. Note that for each blockage, lowerleft corner shares the same $x$ coordinate as upperleft corner and lowerright corner shares the same $x$ coordinate as upperright corner. We only need to sort left boundary and right boundary segment of each blockage instead of point by point to speed up sorting process. Note that a fundamental operation of sweep line algorithm is to keep an active set $A$ of $v$ such that all points in $A$ are visible to $v$. We thus build an active set $A$ and dynamically keep $A$ by adding and deleting points for set $A$. Starting with an empty set $A$, we check each point $v_{i}$ in the sorted list $V_{s s}$. If $v_{i}$ is not a lowerleft corner of a blockage (i.e., $v$ is either a pin or one of the other three corners of a blockage), we just add $v_{i}$ into set $A$, otherwise we perform edge connection for $v_{i}$ as follows. Suppose $v_{i}$ is the lowerleft corner of blockage $b_{j}$. We first pick the point $v_{i+1}$ which is right after $v_{i}$ in the list of $V_{s s}$. Note that $v_{i+1}$ must be the upperleft corner point of $b_{j}$ due to the attribute of our sorted list $V_{s s}$. Then, we check each point $a$ in $A$. If point $a$ lies in region $R_{2}$ of $b_{j}$, we add this point to another set $A_{s}$. If $b_{j}$ is not a vertical blockage, we delete this point from $A$. After that, we find out two points $q$ and $q^{\prime}$ from $A_{s}$ which are closest to $v_{i}$ and $v_{i+1}$, respectively, in rectilinear distance. Finally, we add two edges, $\left(v_{i}, q\right)$ and $\left(v_{i+1}, q^{\prime}\right)$, into graph $G$. Note that $q$ and $q^{\prime}$ could be the same point in $A_{s}$. Before we move to the next point in $V_{s s}$, we vacate set $A_{s}$.

An example is shown in Figure 10. The bold-faced segments are the edges added to graph $G$ after edge connection is performed for $R_{2}$ of all corner points. Since search region $R_{6}$ has the reverse sweep sequence, we can make use of the same sorted list $V_{s s}$ to perform edge connection for $R_{6}$ in Pass one. Similarly, in Pass two, we perform edge connection for search region $R_{4}$ and $R_{8}$ of blockages after sorting $V_{s}$ in a non-decreasing order with their $y$ coordinates. In Pass three, we perform similar edge connection for $R_{1}$ and $R_{5}$ of blockages and $R_{1}$ and $R_{3}$ of pins after sorting $V_{s}$ in non-decreasing $x+y$. Similarly, in Pass four, edge connection is performed for search region $R_{3}$ and $R_{7}$ of blockages and $R_{2}$ and $R_{4}$ of pins after $V_{s}$ is sorted in non-decreasing $y-x$.

In order to achieve $O(n)$ running time, the active sets $A$ and $A_{s}$ must be efficiently maintained so that searching, deletion, and insertion each can be done in $O(\log n)$ time. The spanning graph after four passes is shown in Figure 11. The algorithm of spanning graph construction in RSMTRB is summarized in Algorithm 1. The detail procedure of edge connection for search region $R_{2}$ is summa-

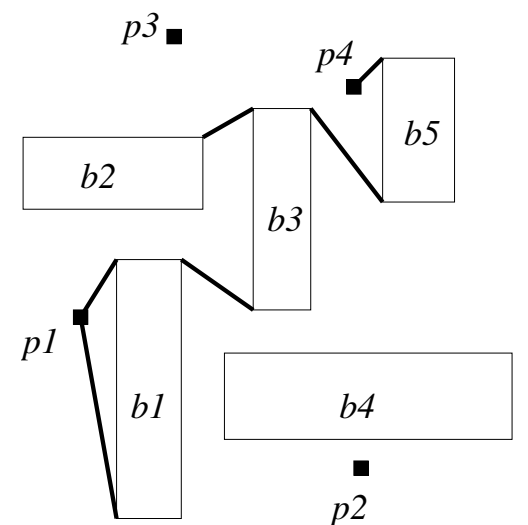

Figure 10: Edge connection for region $R_{2}$.

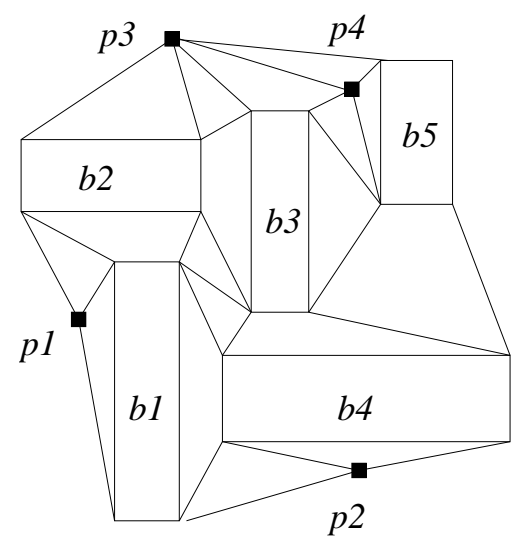

Figure 11: Complete spanning graph

rized in Algorithm 2.

\author{
Algorithm 1: Spanning graph construction in $R S M T R B$ \\ Input: $V_{s}$ \\ sort $V_{s}$ by non-decreasing $x$; \\ perform edge connection for $R_{2}$ and $R_{6}$ of all corners \\ sort $V_{s}$ by non-decreasing $y$; \\ perform edge connection for $R_{4}$ and $R_{8}$ of all corners; \\ sort $V_{s}$ by non-decreasing $x+y$; \\ perform edge connection for $R_{1}$ and $R_{5}$ \\ of all corners and $R_{1}$ and $R_{3}$ of all pins; \\ sort $V_{s}$ by non-decreasing $y-x$; \\ perform edge connection for $R_{3}$ and $R_{7}$ \\ of all corners and $R_{2}$ and $R_{4}$ of all pins; \\ Return:spanning graph $G$ for $V_{s}$
}

\section{5. $R S M T$ CONSTRUCTION}

After we complete the spanning graph $G=(V, E)$, we apply a heuristic to construct an $R S M T$ based on the graph $G$. The heuristic consists of following six steps. An example is shown from Figure 12 to Figure 17 to illustrate each step.

Step 1: Let $P$ be a set of pins for a net. We construct a complete undirected graph $G_{1}=\left(V_{1}, E_{1}\right)$ from $G$ and $P$ in such a way that $V_{1}=P$ and for each edge $\left(v_{i}, v_{j}\right) \in E_{1}$, the length on the edge $\left(v_{i}, v_{j}\right)$ is equal to the length of the shortest path from $v_{i}$ to $v_{j}$ in graph $G$. See Figure 12 as an illustration for step 1. The length of edge $\left(p_{1}, p_{3}\right)$ in $G_{1}$ is the length of the shortest path from $p_{1}$ to $p_{3}$ 


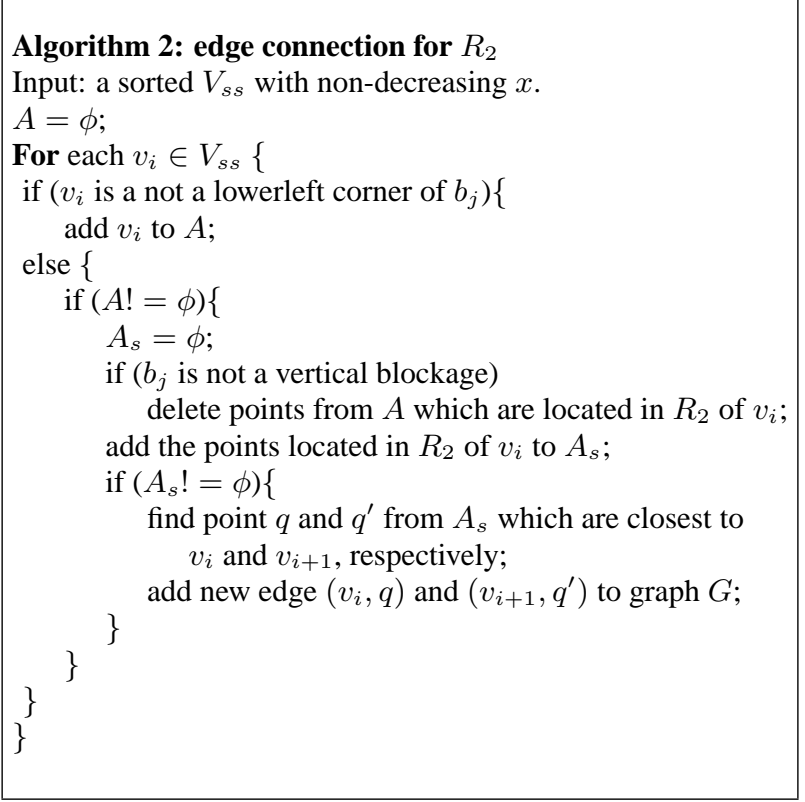

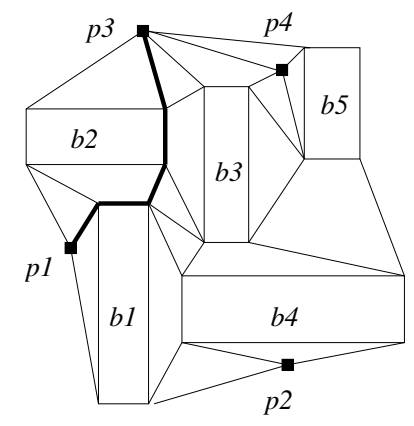

$G$

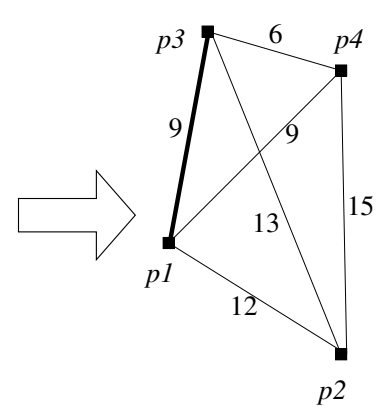

G1
Figure 12: Step 1

in graph $G$, which is shown in bold-fased segments.

Step 2: Find the minimum spanning tree $T_{1}$ of $G_{1}$. If there exist several minimum spanning trees, pick an arbitrary one. In practice, we always pick the first one which we obtain. See Figure 13 as an illustration for step 2.

Step 3: Construct the subgraph $G_{s}$ of $G$ as follows. First we construct an intermediate subgraph $G_{s}^{\prime}=\left(E^{\prime}, V^{\prime}\right)$ of $G$ by replacing each edge in $T_{1}$ by its corresponding shortest path in $G$. If there are several shortest paths, pick an arbitrary one. In practice, we always pick the first one which we obtain. The edges of resulting graph $G_{s}^{\prime}$ are shown as bold-faced solid segments in Figure 14. Then we build $G_{s}$ by adding edge $\left(v_{i}, v_{j}\right)$ to $G_{s}^{\prime}$, where $v_{i} \in V^{\prime}, v_{j} \in V^{\prime}$ and $\left(v_{i}, v_{j}\right) \in E$. The added edges are shown as bold-faced dashed segments in Figure 14.

Step 4: Find minimum spanning tree $T_{s}$ of $G_{s}$. If there are several minimum spanning trees, pick an arbitrary one. See Figure 15 as an illustration for step 4.

Step 5: Construct a Steiner tree $T_{h}$ from $T_{s}$ by deleting edges in $T_{s}$, if necessary, so that all the leaves in $T_{h}$ are pins. As illustrated in Figure 16, in this example, we could not find any leaf which is blockage corner point.

Step 6: Rectilinearize $T_{h}$ to obtain a rectilinear steiner tree $T_{r}$. See Figure 17 as an illustration for step 6.

\section{EXPERIMENTAL RESULTS}

We implemented the spanning graph based $R S M T R B$ algorithm
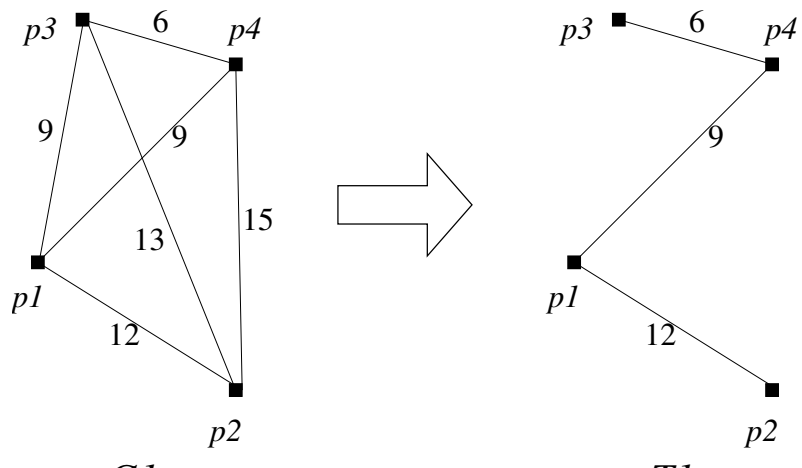

G1

Figure 13: Step 2
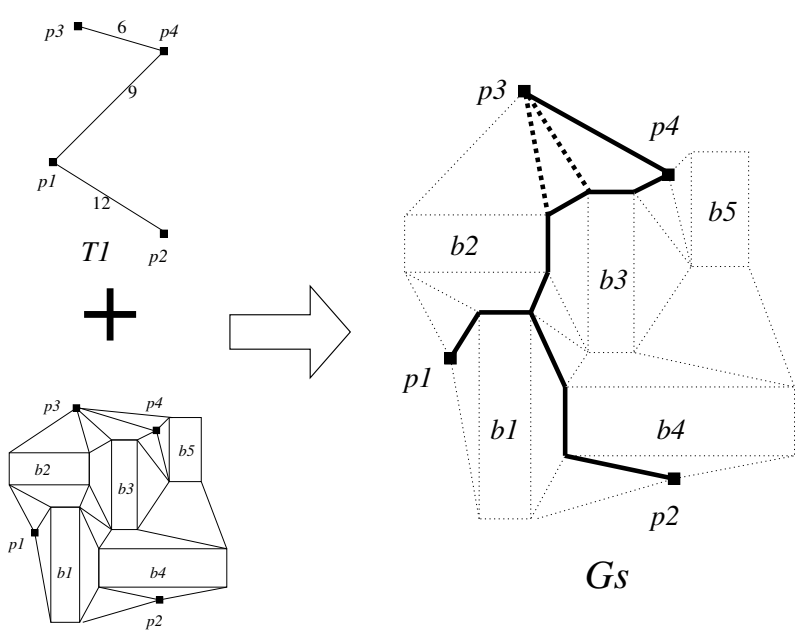

G

Figure 14: Step 3

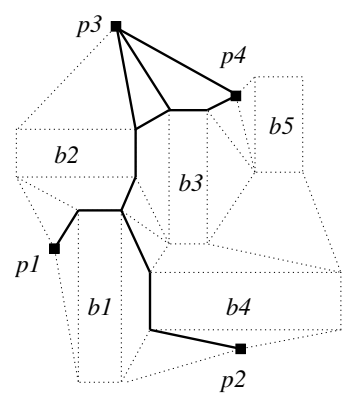

Gs

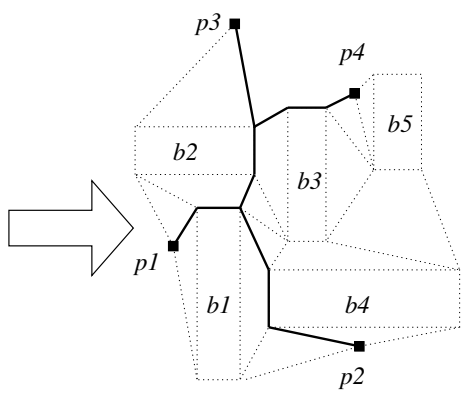

$T s$
Figure 15: Step 4 


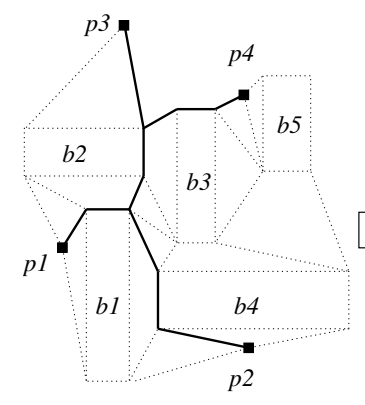

$T s$

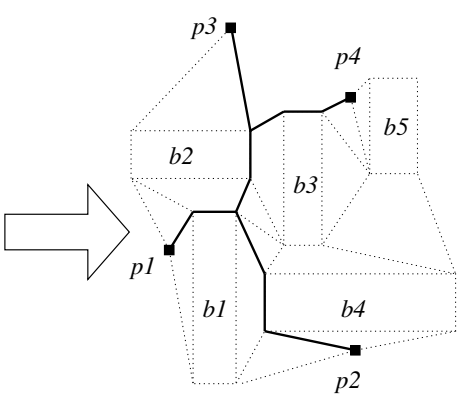

$T h$

Figure 16: Step 5

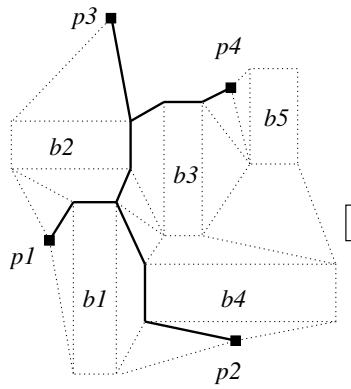

$T s$

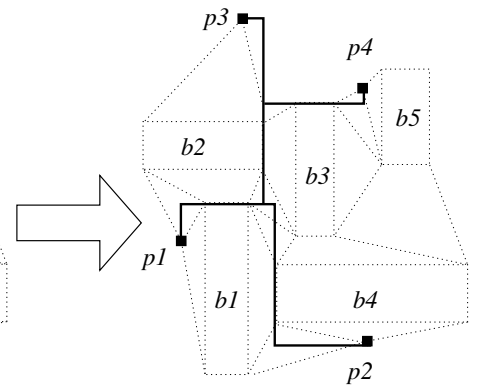

$T h$
Figure 17: Step 6

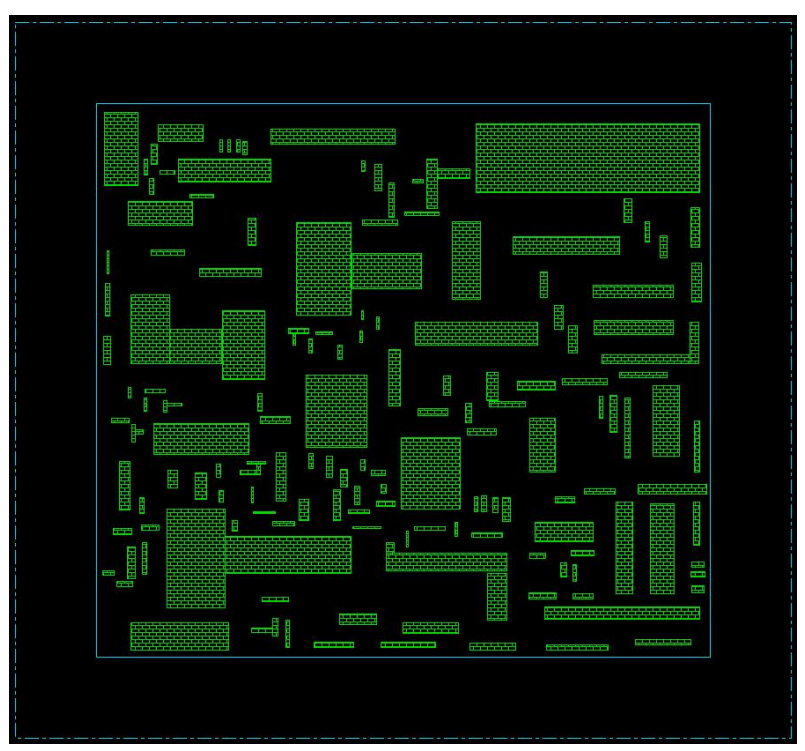

Figure 18: Blockage placement in test case 1 in $\mathrm{C}++$ language. We compile and run the program on Intel Pentium 4 machine with $2.80 \mathrm{GHz}$ frequency and $1.5 \mathrm{~GB}$ RAM. We pick 5 industrial test cases and randomly create blockages for these test cases. In case a source pin is inside a blockage, we move this pin to upperleft corner of this blockage. If sink pin is inside a blockage, we move this pin to lowerright corner of this blockage. The statistic data of test cases are listed in Table 1. The blockage placement for test case 1 is shown in Figure 18 as an example. We compare our approach with the traditional sequential approach illustrated in Figure 1.

The total wire length and run time comparison is given in Table 2. The results show that our spanning graph based approach can reduce $12.081 \%$ (on average) wire length of $R S M T$, comparing to sequential approach. And the run time is only increased by $48.440 \%$ on average.

Table 3 shows the wire length reduction percentage of different pin nets for each test case. It is not hard to find that our approach has most significant wire length reduction on multi-pin nets, especially while the pin number exceeds 50 . This will benefit timing closure for the whole design. Since in general, multi-pin nets or high fanout nets contribute most significant portion of interconnect delay in the most timing critical path of a circuit.

\begin{tabular}{|c|c|c|c|c|c|}
\hline Test cases & 1 & 2 & 3 & 4 & 5 \\
\hline num of inst & 158672 & 35601 & 437444 & 277356 & 450367 \\
\hline num of I/O pins & 865 & 201 & 1774 & 1453 & 1276 \\
\hline num of nets & 169243 & 36244 & 477380 & 285556 & 451250 \\
\hline 2 pin net (\%) & 52.2 & 63.4 & 57.4 & 71.4 & 76.1 \\
\hline $3-10$ pin net (\%) & 42.9 & 32.8 & 21.2 & 16.3 & 16.8 \\
\hline $11-50$ pin net (\%) & 4.5 & 3.2 & 17.6 & 10.1 & 6.7 \\
\hline $51-100$ pin net(\%) & 0.34 & 0.47 & 3.50 & 2.01 & 0.36 \\
\hline$\geq 101$ pin net(\%) & 0.06 & 0.13 & 0.30 & 0.20 & 0.03 \\
\hline num of blkgs & 145 & 37 & 136 & 539 & 487 \\
\hline
\end{tabular}

Table 1: Statistics of test cases

\section{CONCLUSION AND DISCUSSION}

In this paper, we propose an efficient and effective approach to construct rectilinear steiner minimum tree with rectilinear blockages. The connection graph we used in this approach is called spanning graph which only contains $O(n)$ edges and vertices. An $O(n \log n)$ time algorithm is proposed to construct spanning graph for $R S M T R B$. The experimental results shows that this approach can achieve a solution with significantly reduced wire length. The total run time increased is negligible in the whole design flow.

Since our heuristic is graph-based, it can be easily modified to handle other metrics. A possible extension is to construct timing aware routing tree topology for a net among routing blockages.

\section{REFERENCES}

[1] P. Berman, U. Fossmeier, M. Kaufmann, M. Karpinski and A. Zelikovsky. Approaching the 5/4-approximation for rectilinear Steiner trees. Proc. European Symp. on Algorithms (ESA), Springer Verlag Lecture Notes in Computer Science 762, pages 533-542, 1994.

[2] M. Borah, R. M. Owens, and M. J. Irwin. A fast and simple Steiner routing heuristic. , Springer Verlag Lecture Notes in Computer Science 762, pages 533-542, 1994.

[3] M. Hanan. On Steiner's problem with rectilinear distance. SIAM Journal on Applied Mathematics, 14, pages 255-265, 1966.

[4] F. K. Hwang and D. S. Richards and P. Winter. The Steiner tree problem. North-Holland, annals of Discrete Mathematics 53, 1992.

[5] A. B. Kahng and G. Robins. A new class of iterative Steiner tree heuristics with good performance. IEEE Transaction on CAD, 11, pages 1462-1465, 1992.

[6] A. Zelikovsky. An 11/6-approximation for the network Steiner tree problem. Algorithmica, 9, pages 463-470, 1993. 
[7] M. Garey and D. Johnson. The rectilinear Steiner tree problem is NP-complete. SIAM J. Appl. Math,32, pages 826-834, 1977.

[8] C. Y. Lee. An algorithm for path connections and its application. IRE Transaction on Electronic Computers,V.EC-10, pages 346-365, 1961.

[9] S. B. Akers. A modification of Lee's Path Connection Algorithm. IEEE Transaction on Electronic Computer,16(4), pages 97-98, 1967.

[10] J. Soukup. Fast Maze Router. Proceeding. of 15th Design Automation Conference, pages 100-102, 1978.

[11] F. Rubin. The Lee Connection Algorithm. IEEE Transaction on Computer, 23, pages 907-914, 1974.

[12] Y. Yang, Q. Zhu, T. Jing, X. Hong and Y. Wang. Rectilinear Steiner minimal tree among obstacles. ASIC 5th Intl. Conf., Vol. 1, 21-24 pages 348-351, Oct. 2003.

[13] T. Lengauer. combinatorial Algorithms for Integrated Circuit Layout. Wiley, England, 1990.

[14] J. Ganley and J. P. Cohoon. Routing a Multi-Terminal Critical Net: Steiner Tree Construction in the Presence of Obstacles. Intl. Symp. on Circuits and Systems, Vol. 1, pages 113-116, 1994.

[15] K. L. Clarkson, S. Kapoor and P. M. Vaidya. Rectilinear shortest paths through polygonal obstacles in $O\left(n \log ^{2} n\right)$ time. ACM Symp. on Computational Geometry, pages 251-257, 1987.

[16] K. L. Clarkson, S. Kapoor and P. M. Vaidya. Rectilinear shortest paths through polygonal obstacles in $O\left(n \log ^{3 / 2} n\right)$ time. Unpublished manuscript.

[17] H. Zhou, N. Shenoy, and W. Nicholls. Efficient spanning tree construction without delaney triangulation. Information Processing Letter, 81(5), 2002.

\begin{tabular}{|c|c|c|c|c|c|c|}
\hline \hline & \multicolumn{3}{|c|}{ Total wire length $(\mu \mathrm{m})$} & \multicolumn{3}{c|}{ Total run time $(s)$} \\
\hline Test case & Sequential & Ours & decreased $(\%)$ & Sequential & Ours & increased $(\%)$ \\
\hline 1 & 15174595 & 13855164 & 8.695 & 9.531 & 12.193 & 27.926 \\
\hline 2 & 1995 & 1820 & 8.778 & 1.404 & 2.361 & 68.112 \\
\hline 3 & 2854122 & 2400499 & 15.890 & 18.671 & 27.339 & 46.425 \\
\hline 4 & 2452867 & 2051254 & 16.371 & 14.998 & 20.668 & 37.805 \\
\hline 5 & 1022723 & 913584 & 10.670 & 13.576 & 21.984 & 61.933 \\
\hline \multicolumn{3}{|c|}{ On average } & 12.081 & & & 48.440 \\
\hline
\end{tabular}

Table 2: Totoal wire length and run time comparison.

\begin{tabular}{|c|c|c|c|c|c|}
\hline \hline Test case & 2 pin net(\%) & $3-10$ pin net(\%) & $11-50$ pin net (\%) & $51-100$ pin net (\%) & $\geq 101$ pin net (\%) \\
\hline 1 & 4.366 & 14.042 & 15.301 & 22.923 & 29.989 \\
\hline 2 & 4.619 & 13.990 & 14.935 & 23.494 & 30.492 \\
\hline 3 & 6.984 & 19.831 & 21.404 & 27.230 & 32.103 \\
\hline 4 & 6.972 & 19.983 & 22.398 & 24.506 & 33.840 \\
\hline 5 & 4.581 & 15.720 & 18.591 & 20.383 & 31.729 \\
\hline On average & 5.492 & 16.713 & 18.526 & 23.707 & 31.631 \\
\hline
\end{tabular}

Table 3: Wirelength reduction percentage on nets. 